\title{
Retraction Note to: Identification of genes associated with the differentiation potential of adipose-derived stem cells to osteocytes or myocytes
}

Yizhong Ren ${ }^{1}$ Changxu Han ${ }^{2} \cdot$ Jingjuan Wang ${ }^{2} \cdot$ Yanbo Jia $^{2} \cdot$ Lingyue Kong $^{2}$.

Tu Eerdun ${ }^{2} \cdot$ Lishuan $\mathrm{Wu}^{3} \cdot$ Dianming Jiang ${ }^{1}$

Published online: 17 August 2015

(c) Springer Science+Business Media New York 2015

Retraction Note to: Mol Cell Biochem (2015)

400:135-144

DOI 10.1007/s11010-014-2269-y

The Publisher and Editor retract this article in accordance with the recommendations of the Committee on Publication Ethics (COPE). After a thorough investigation we have strong reason to believe that the peer review process was compromised.

The online version of the original article can be found under doi:10.1007/s11010-014-2269-y.

Dianming Jiang

DianmingJiangdmj@163.com

1 Department of Orthopaedics, The First Hospital of Chongqing Medical University, No. 1 Youyi Road, Yuzhong District, Chongqing City 400016, China

2 Department of Arthroscopy and Sports Medicine, The Second Affiliated Hospital of Inner Mongolia Medical University, No. 1 Yingfang Street, Hyumin District, Huhehot 010055, Inner Mongolia, China

3 Department of Anesthesiology, The Second Hospital of Inner Mongolia Medical University, No. 1 Yingfang Street, Hyumin District, Huhehot 010055, Inner Mongolia, China 